\title{
What matters in the cued task-switching paradigm: Tasks or cues?
}

\author{
ULRICH MAYR \\ University of Oregon, Eugene, Oregon
}

\begin{abstract}
Schneider and Logan (2006) recently showed that cue-switch and task-switch costs are sensitive to the relative probability of cue switches versus task switches. From this they concluded that taskswitch costs reflect priming of cue-cue transitions rather than actual task-switching operations. However, because this design confounded probability of specific cue transitions with probability of task switches, the results could also reflect task-switch-level adjustments. The present experiment $(N=80)$ pits the critical prediction of the cue-priming account, namely that costs for high-probability cue-cue transitions are smaller than for low-probability cue-cue transitions, against the main prediction of the switch-probability account, namely that switch probability, irrespective of specific cue-cue transitions, determines switch costs. Whereas the cue-priming prediction was rejected, a specific version of the probability account-that subjects are sensitive to the probability of a task switch, given a cue switch-was fully confirmed. Thus, tasks are in fact the critical representational units that determine task-switch cost.
\end{abstract}

Switch costs as assessed in the task-switching paradigm are typically interpreted as a reflection of the processing demands involved in changing task-specific cognitive configurations (for a recent review, see Monsell, 2003). Recently, however, for one variant of the task-switching paradigm, the so-called cuing paradigm, this interpretation of switch costs has been called into question. In the cuing paradigm, tasks vary randomly on a trial-by-trial basis and each task is indicated by a cue, usually visual. The cuing paradigm allows tight control over when subjects can start preparing for an upcoming task and therefore has proven useful for examining how proactive control influences switch costs (see, e.g., Meiran, 1996). However, in two parallel papers, Logan and Bundesen (2003) and Mayr and Kliegl (2003) noted that, in this paradigm, task changes are completely confounded with cue changes. They therefore introduced a new variant using a 4:2 mapping between cues and tasks. This allows three types of transitions to be examined: no switch, cue switch without task switch, and task switch. The contrast between the no-switch and the cue-switch conditions yields the costs associated with changing the cue (henceforth referred to as cue-switch costs). In both Logan and Bundesen (L\&B) and Mayr and Kliegl (M\&K) these costs were surprisingly large, suggesting that a substantial component of the traditional switch costs had nothing to do with changing cognitive configurations per se. Where the results of $M \& K$ and L\&B differed was with respect to the second contrast, that between the cue-switch and the task-switch transi-

Correspondence concerning this article should be addressed to U. Mayr, Department of Psychology, University of Oregon, Eugene, OR 97403 (e-mail: mayr@darkwing.uoregon.edu). tions (henceforth called true task-switch cost). Whereas this component was substantial in M\&K, it was nonexistent in L\&B; in some of the succeeding papers by Logan and coworkers, the true task-switch costs were present, but only very small (see, e.g., Logan \& Bundesen, 2004). On the basis of the finding of substantial task-switch costs, M\&K concluded that there is true task switching going in the cuing paradigm, whereas L\&B suggested that subjects form compounds between cues and stimuli that allow disambiguating response selection. According to this view, switching occurs between these compounds and not between the actual tasks. Whether or not there is true task switching in the cued task-switching paradigm is a question of considerable theoretical relevance: At stake is to what degree task sets are a mandatory, organizing principle in human action control.

One noteworthy difference between the procedure used by L\&B and the one used by M\&K was in switch probabilities. In L\&B, all cues and stimuli were selected randomly without constraints. With four cues mapped to two tasks, there were four different no-switch cue-cue transitions and four different cue-switch cue-cue transitions, but eight different possible task-switch cue-cue transitions (see Table 1 for a listing of all specific cue transitions possible with a 4:2 cue-task mapping). Thus, random cue selection led to a no-switch and a cue-switch probability of .25 each, but a task-switch probability of .5 . In contrast, $M \& \mathrm{~K}$ had used a probability of .33 for each type of transition (see also Experiment 3 in Monsell \& Mizon, 2006). Why should this matter? A critical decision that subjects may have to make on each trial of the cued task-switching paradigm is whether or not to abandon the current task set. It is possible that this decision is at least partly based on the switch probability in a given situation. 
Table 1

Design of the Present Experiment With Specific Cue Transitions, Probabilities of Transition Types, and Conditional Probabilities of Task Switches, Given Cue Switches for the Two Between-Subjects Groups (High vs. Low Probability Task-Switch-2 Transitions)

\begin{tabular}{llcccc}
\hline & & \multicolumn{3}{c}{ Transition Probabilities } \\
\cline { 3 - 5 } \multicolumn{1}{c}{ Sransition Type } & Cue-Cue Transitions & High & Low & M\&K & L\&B \\
\cline { 3 - 5 } No-switch & A-A, B-B, C-C, D-D & .2 & .3 & .33 & .25 \\
Cue-switch & A-B, B-A, C-D, D-C & .2 & .3 & .33 & .25 \\
Task-switch-1 & A-C, B-D, C-B, D-A & .2 & .3 & .33 & .25 \\
Task-switch-2 & A-D, B-C, C-A, D-B & .4 & .1 & 0 & .25 \\
P(Task switch/cue switch) & & .75 & .57 & .5 & .66 \\
\hline
\end{tabular}

Note-Specific transitions for task-switch-1 and task-switch-2 for the present experiment were counterbalanced across subjects. Switch probability (high vs. low) was manipulated between subjects. Cues A and B are associated with Task 1; Cues C and D are associated with Task 2. In the actual experiment reported here, semantic task labels were used. For comparison, corresponding transition probabilities used by Mayr and Kliegl (2003; M\&K) and Logan and Bundesen (2003; L\&B) are shown.

We will refer to such a switch-probability-based adaptation of configuration activities simply as a task-level adaptation account (for a nearly identical suggestion, see Monsell \& Mizon, 2006). This type of adaptation might occur in two different ways. First, relatively high switch probabilities could lead subjects to generally prepare for the more difficult task-switch transitions by abandoning or suppressing tasks after each trial (or on a majority of trials). Whereas this would be advantageous if a task change actually occurred on a given trial, this strategy would lead to costs on trials where the task stayed the same, whether these trials involved a cue switch or not. The net result would be a reduction of switch costs, which would go along with an increase in both no-switch and cue-switch response times (RTs). A second possibility is that subjects might also take some cue-based information into account when deciding whether or not to abandon the current task set: Specifically, the result of a fast check of the extent to which the cue has changed relative to the preceding trial might influence their decisions. Thus, what counts here is the conditional probability of a task switch, given a cue switch. Whereas in M\&K this conditional probability was .5 , it was .67 in L\&B. The higher conditional probability might bias subjects to generally initiate a task switch in case of a cue switch. This would reduce switch costs relative to cue-switch RTs, but it would not necessarily lead to an increase of no-switch RTs. In other words, if subjects operate on the basis of conditional probabilities, we might see a reduction of true task-switch costs and an increase of cue-switch costs as a function of an increase of taskswitch probability.

Monsell and Mizon (2006) and Schneider and Logan (2006) recently reported experiments in which both cue and task transition probabilities were manipulated. For example, in Schneider and Logan (2006), the probability of no-switch, cue-switch, and task-switch transitions was varied across three different groups in such a way that for each group one of the three transitions was $p=.7$ and the other two were $p=.15$. Results showed that these probability manipulations did in fact have strong effects on the pattern of cue-switch and task-switch costs. Specifically, true taskswitch costs were very small when task-switch transitions occurred with high probability, cue-switch costs were very large when no-switch costs occurred with high probability (while true task-switch costs were substantial), and both types of costs were moderate when cue-switch transitions were highly probable. Generally, very similar results were also reported by Monsell and Mizon. Although this overall pattern is consistent with the task-level adaptation account (and was also interpreted in this manner by Monsell \& Mizon, 2006), Schneider and Logan (2006) proposed a very different interpretation. Extending the L\&B cuepriming model, they suggested that subjects learn highprobability, specific cue-cue transitions. According to this view, task-switch costs are reduced in case of highprobability switch transitions, not because subjects adapt to frequent switching per se. Rather, reduced switch costs result from the greater probability of the specific cue-cue transitions that go along with a task switch, if task-switch probability is high. For example, if Cues A and B indicate Task 1 and Cues $\mathrm{C}$ and $\mathrm{D}$ indicate Task 2, the cue-cue transitions $\mathrm{A}-\mathrm{C}, \mathrm{A}-\mathrm{D}, \mathrm{C}-\mathrm{A}$, and $\mathrm{C}-\mathrm{B}$ would have a higher probability than all other possible transitions. Of course, the same explanation also holds for the reduced cue-switch costs, in the case of frequent cue-switch trials, or reduced no-switch RTs, in the case of frequent no-switch trials. Clearly, this account can explain the results of both Schneider and Logan (2006) and Monsell and Mizon just as well as the task-level adaptation account can.

Obviously, the simple manipulation of task-switch probabilities (as used in Monsell \& Mizon, 2006, and Schneider \& Logan, 2006) confounds switch probability and the probability of specific cue-cue transitions and therefore does not allow an emperical distinction between task-level adaptation and the cue-priming account. However, the two accounts would make different predictions if we managed to manipulate the probability of specific cue transitions. The design used in the present study capi- 
talizes on the previously mentioned fact that, with a $4: 2$ mapping between cues and tasks, there are eight different cue-cue transitions for task switches but only four for cue-switch and no-switch transitions. This allowed us to expose subjects to identical cue-cue transition frequencies for the no-switch, the cue-switch, and one half of the possible task-switch transitions (henceforth referred to as task-switch-1 transitions). For the second half of the possible task-switch transitions (henceforth referred to as task-switch-2 transitions) we used a between-groups manipulation, where one group was exposed to a low probability and the other group to a high probability of task-switch-2 transitions (see Table 1). This manipulation allowed two independent critical tests of the task-level adaptation versus the cue-priming account.

First, if the task-level adaptation account has any merit, the manipulation of task-switch-2 probability should affect task switch and possibly also cue switch costs for the other three transition types (i.e., no-switch vs. cue-switch vs. task-switch-1). Specifically, when the probability of task-switch-2 transitions is high, the unspecific switch probability also increases. This in turn may induce subjects to adapt either to the generally increased switch probability or to the increased conditional probability of task switches, given a cue switch. Thus, this leads to the prediction of smaller task-switch costs (computed as the difference between cue-switch and task-switch-1 transitions) and possibly also larger cue-switch costs (computed as the difference between cue-switch and no-switch transitions) than when task-switch-2 transitions are frequent. It is important to note that we will only look at task-switch-1 transitions, to examine task-switch costs. Thus, any effect on task-switch costs could not be explained in terms of probabilities of specific cue-cue transitions, because these are identical across the two groups of subjects and three transition types (no-switch, cue-switch, and taskswitch-1 transitions). ${ }^{1}$

Second, the manipulation of task-switch-2 probabilities allows us to examine a straightforward prediction of the cue-priming model: that frequent cue-cue transitions should be performed faster than infrequent cue-cue transitions. We therefore need to compare task-switch-1 with task-switch-2 transitions in both groups.

To further examine the characteristics of possible general adaptation effects, we also manipulated the cuestimulus interval (CSI). On the one hand, general adaptation effects might affect processes that with sufficient time can be executed proactively - that is, before the stimulus appears. In this case, they would fully affect cue-switch or task-switch costs only during a short CSI but would be absorbed into the preparation interval if the CSI were long. On the other hand, adaptation effects might affect the availability of the just-executed task in a manner less affected by proactive processes. For example, general adaptation effects might operate through the degree to which the just-executed task-set is inhibited. Task-set inhibition has been shown to exert effects that are relatively insensitive to preparatory activity (see, e.g., Mayr \& Keele, 2000).

\section{METHOD}

\section{Participants}

Eighty students at the University of Oregon participated in a 1-h session in exchange for course credits.

\section{Task, Stimuli, and Design}

Depending on the task, participants were supposed to distinguish either the color or the shape of an object. The object could be a circle, a square, or a triangle of about the same size (e.g., the side length of the square was $1 \mathrm{~cm}$ ), and it could be green, blue, or red. The object was presented on a black background within a white frame with a side length of $2.5 \mathrm{~cm}$. The frame was visible throughout the entire block. Responses were entered with the index, middle, or ring finger of the preferred hand, using the $1 \mathrm{key}$, the $2 \mathrm{key}$, and the 3 key of the numerical keyboard. Circle and green were mapped onto the $1 \mathrm{key}$, square and blue were mapped onto the $2 \mathrm{key}$, and triangle and red were mapped onto the $3 \mathrm{key}$. Stimuli remained on the screen until the correct response was entered. Thus, in case of an incorrect response, participants could continue only after pressing the correct response.

The interval between the response and the stimulus for the next trials was $1,100 \mathrm{msec}$. The CSI was either 100 or $1,000 \mathrm{msec}$. The cue for the color task was either the word color or the word $d y e$. The cue for the shape task was either the word shape or the word form.

With two cues per task, there are four possible cue transitions for no-switch and cue-switch trials and eight possible transitions for task-switch trials. The eight cue transitions for task-switch trials were assigned to two sets of four (Set 1, color-shape, dye-form, shape-dye, form-color; Set 2, color-form, dye-shape, shape-color, form-dye). Subjects were randomly assigned to two groups. In both groups, one of the two sets was presented, with equal frequency, as a no-switch transition and as a cue-switch transition. In the highswitch-probability group, the remaining set of task-switch cue transitions occurred with high probability; in the low-switch-probability group it occurred with low probability. Table 1 shows the exact frequencies for the two groups.

\section{Procedure and Design}

Block length was 90 trials. There were two practice blocks in which participants could become familiar with each of the two tasks and the cue-task associations. The order of introducing the two different tasks was counterbalanced. Following practice, eight test blocks were presented. Blocks alternated between short CSI and long CSI, with the CSI of the initial test block counterbalanced across participants.

\section{RESULTS AND DISCUSSION}

RTs longer than 4,000 msec were excluded from analysis (eliminating 1\% of the RT distribution), as were RTs from incorrect trials or from trials following incorrect trials.

Figure 1 shows cue-switch and task-switch costs as a function of probability of task-switch-2 transitions. Two separate $2 \times 2 \times 2$ ANOVAs were conducted, one contrasting no-switch and cue-switch transitions, the other cue-switch and task-switch transitions. Both analyses also included the group factor (high- vs. low-probability taskswitch-2 transitions) and the CSI factor. As can be seen, irrespective of probability of task-switch-2 transitions, there were substantial cue-switch costs $[F(1,78)=58.36, p<$ $.001]$, which became smaller for the long CSI condition $[F(1,78)=10.36, p<.01]$. There were also substantial true task-switch costs in all conditions $[F(1,78)=152.96$, 


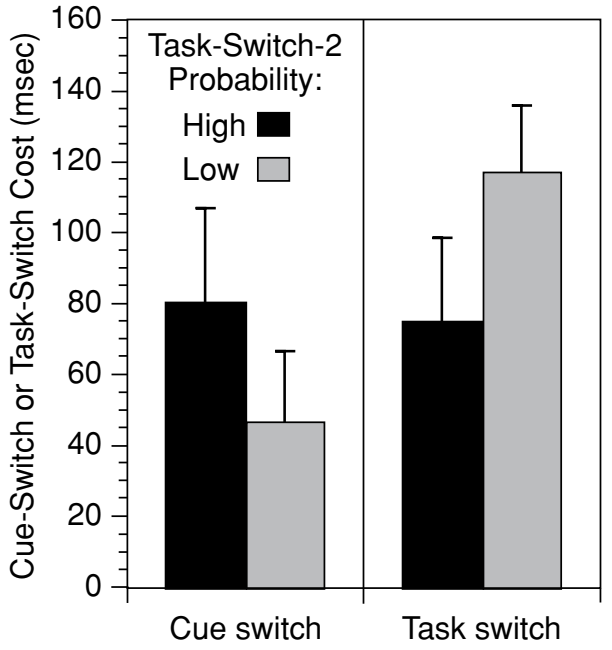

Figure 1. Cue-switch and task-switch costs as a function of high versus low probability of task-switch-2 transitions. Error bars represent $95 \%$ between-subjects confidence intervals.

$p<.01]$, which were stable across the CSI manipulation $[F(1,78)=.53, p>.4]$. This pattern of CSI-dependent cue-switch and CSI-independent true task-switch costs replicates the results reported by Mayr and Kliegl (2003; but see Monsell \& Mizon, 2006).

For the critical question regarding general adaptation effects, we need to look at the effect of the probability of task-switch-2 transitions on the remaining transitions. As is apparent from Figure 1, cue-switch costs were larger when task-switch-2 transitions were frequent, whereas task-switch costs were larger when task-switch-2 transitions were rare. Both the interaction between group and the cue-switch factor $[F(1,78)=4.15, p<.05]$ and the interaction between group and the task-switch factor $[F(1,78)=8.52, p<.01]$ were reliable. With regard to errors, there were no effects that counteracted RT re- sults. True task-switch costs were smaller for frequent task-switch-2 transitions than for the rare task-switch-2 transitions $[F(1,78)=5.05, p<.05]$. No comparable effect was observed for cue-switch costs $[F(1,78)=0.0]$. Overall, the prediction that switch probability modulates costs independently of specific cue transitions was fully confirmed.

Next, we turn to the critical prediction of the cue-priming account that can be tested in our design - namely, that high-probability cue-cue transitions (i.e., task-switch-1 for the task-switch-2 rare group, and task-switch-2 for the task-switch-2 frequent group) are processed faster than low-probability cue-cue transitions. Table 2 contains the relevant results in the form of difference scores (frequency effects). As is apparent, three of the four RT scores were slightly negative (indicating faster processing for the lowprobability transitions) and three of the four error scores were slightly negative (indicating more accurate processing of low-probability transitions). Neither of the two positive scores $[t(39)>.24, p>.8]$ was reliable; nor were any of the negative scores [t(39) $>1.7, p>.09]$. Possibly, specific cue-transition learning effects can be found only later, in practice. However, an analysis that focused on the second half of the test blocks again revealed no trace of cue-specific learning. Thus, the prediction that probability of specific cue-cue transitions would modulate switch costs could not be confirmed in the present data set.

\section{CONCLUSION}

The results presented here suggest that subjects modulate their switching behavior as a function of switch probability. More precisely, the joint increase of cue-switch costs and decrease of task-switch costs as a function of switch probability suggests that subjects were responding to the conditional probability of a task switch, given a cue switch. It seems plausible that people can conduct a fast same-different judgment regarding whether or not the

Table 2

Mean RTs and Error Scores (With $S D$ s) as a Function of Between-Subjects Groups (High vs. Low Task-Switch-2 Probability), CSI, and All Relevant Transition Types

\begin{tabular}{|c|c|c|c|c|c|c|c|c|c|}
\hline \multirow[b]{3}{*}{ CSI } & \multirow[b]{3}{*}{ Transition Type } & \multicolumn{8}{|c|}{ Task-Switch-2 Probability } \\
\hline & & \multicolumn{4}{|c|}{ High } & \multicolumn{4}{|c|}{ Low } \\
\hline & & RT & $S D$ & Err. & $S D$ & RT & $S D$ & Err. & $S D$ \\
\hline \multirow[t]{5}{*}{$100 \mathrm{msec}$} & No-switch & 1,039 & 178 & 4.2 & 3.5 & 971 & 216 & 5.8 & 4.9 \\
\hline & Cue-switch & 1,136 & 228 & 3.8 & 3.5 & 1,041 & 225 & 4.8 & 5.4 \\
\hline & Task-switch-1 & 1,208 & 220 & 7.3 & 4.8 & 1,148 & 244 & 9.8 & 7.3 \\
\hline & Task-switch-2 & 1,210 & 226 & 7.8 & 5.6 & 1,153 & 275 & 9.4 & 7.9 \\
\hline & Frequency effect & -2 & 63 & -.5 & 4.0 & 5 & 121 & -.4 & 4.8 \\
\hline \multirow[t]{5}{*}{$1,000 \mathrm{msec}$} & No-switch & 829 & 173 & 5.1 & 3.8 & 812 & 204 & 6.1 & 6.0 \\
\hline & Cue-switch & 893 & 182 & 3.8 & 3.4 & 836 & 217 & 5.4 & 4.8 \\
\hline & Task-switch-1 & 966 & 191 & 7.0 & 4.7 & 962 & 240 & 10.6 & 7.5 \\
\hline & Task-switch-2 & 978 & 192 & 7.7 & 5.0 & 938 & 254 & 10.8 & 1.0 \\
\hline & Frequency effect & -12 & 84 & -.7 & 3.7 & -24 & 90 & .1 & 5.6 \\
\hline
\end{tabular}

Note-Frequency effects are the RT and error difference scores yielded by subtracting highprobability task-switch transitions from low-probability task-switch transitions within the two between-subjects groups. 
cue has changed from the preceding trial. In case of a cue change, and if switch probability is high, subjects may be more inclined to abandon the current task set. Abandoning the last relevant task should result in additional costs on cue-switch trials, because the abandoned task would then have to be reactivated.

Although we did find clear evidence for general probability effects that could not be explained in terms of specific cue-priming effects, we found no positive evidence for learning of specific cue-cue transitions as suggested by Schneider and Logan (2006). Of course, we cannot rule out the possibility that such learning may have played a role in the studies by Schneider and Logan (2006) or Monsell and Mizon (2006). Manipulations of the probabilities of different task-level transition categories were larger in these studies (15\% vs. $70 \%)$ than were the specific cuecue manipulations we achieved here (40\% vs. $20 \%$ and $10 \%$ vs. $30 \%$; see Table 1 ). There is also no theoretical reason why task-level adaptations and learning of cue-cue transitions could not both exist and maybe become apparent under different boundary conditions. However, our results constitute a clear case in which both true task-switch costs and a probability-based modulation of such costs are present, but cannot be explained in terms of specific cue transitions. Also, our manipulations are of about the same size as those implemented across recent studies using the 4:2 cue-task paradigm (see Table 1). At the very least, this casts doubt on the ability of the cue-based priming model to explain the entire range of cued task-switching effects in terms of cue-based priming effects. Our results are, however, compatible with Monsell and Mizon's account of probability modulations of switch costs. An additional advantage of the adaptation account is that it can readily explain the absence of switch costs in L\&B, which had used a conditional switch probability of .67 , in comparison with the finding of substantial switch costs in M\&K, which had used a conditional switch probability of .5.

The finding that task transitions rather than cue-cue transitions seem to matter in terms of adaptation effects is also broadly consistent with a number of results that suggest a functional dissociation between cue-switch and task-switch costs. For example, cue-switch costs seem more sensitive to practice and CSI manipulations than task-switch costs (e.g., M\&K; but see Monsell and Mizon, 2006, for findings suggesting that task-switch costs can also be affected by CSI), whereas effects of task-set inhibition affect the task-switch component (M\&K). Koch (2001) reported results suggesting implicit learning of a sequence of tasks. Following up on this result, Gotler, Meiran, and Tzelgov (2003) used a 2:1 mapping between cues and tasks and showed that the implicit learning occurred on the task and not on the cue level, again pointing to tasks rather than cues as the critical representational entities in cued task-switching situations. Finally, recent results using EEG (Jost, Mayr, \& Rösler, 2006) and fMRI (Brass \& von Cramon, 2002; Bryck, Gordon, \& Mayr, 2004) also suggest a neural-level dissociation between cue-switch and task-switch costs.
Along with the other recent evidence regarding a dissociation between cue- and task-switch costs, the present data are compatible with the view that subjects step through the trials of a cued-task-switching paradigm by selecting among competing, abstract task sets. Likely, task-set reconfiguration and maintenance are both costly processes, so it is not too surprising to find that people adjust their decisions about when to abandon task sets to the probability structure of a given task-selection situation. An important question for further research is what exactly it means to strategically abandon a task set in case of a high frequency of task-switch transitions. One interesting possibility is that, in case of a high switch probability, subjects may be more inclined to inhibit the just-executed task set (Mayr \& Keele, 2000) when cues change across consecutive trials. As a consequence, it would be more difficult to reactivate a just-suppressed task in case of a cue-switch trial, leading to larger cue-switch and smaller task-switch costs (for a related finding, see Mayr \& Bell, 2006). Interestingly, as was found to be the case for task-set inhibition effects (e.g., Mayr \& Keele, 2000), the generaladaptation effects we obtained in this study were not affected by the CSI manipulation. Of course, the possibility that general-adaptation effects are instantiated via task-set inhibition is based on the assumption that inhibition is sensitive to the general selection context and is therefore under some strategic control. Indeed, Philipp and Koch (2006) recently reported evidence that supported this assumption. They found that task-set inhibition was much larger under conditions of high switch probability than under conditions of low switch probability.

\section{REFERENCES}

Brass, M., \& von Cramon, D. Y. (2002). The role of the frontal cortex in task preparation. Cerebral Cortex, 12, 908-914.

Bryck, R. L., Gordon, K. B. C., \& MaYr, U. (2004, April). Neural correlates of cue-switching and task-switching. Poster presented the Cognitive Neuroscience Society Meeting, San Francisco.

Gotler, A., Meiran, N., \& Tzelgov, J. (2003). Nonintentional task set activation: Evidence from implicit task sequence learning. Psychonomic Bulletin \& Review, 10, 890-896.

Jost, K., MAYR, U., \& RöSLER, F. (2006). Is task switching nothing but cue priming? Evidence from ERPs. Manuscript in preparation.

$\mathrm{KoCH}$, I. (2001). Automatic and intentional activation of task sets. Journal of Experimental Psychology: Learning, Memory, \& Cognition, 27, 1474-1486.

Logan, G. D., \& Bundesen, C. (2003). Clever homunculus: Is there an endogenous act of control in the explicit task cuing procedure? Journal of Experimental Psychology: Human Perception \& Performance, 29, 575-599.

Logan, G. D., \& Bundesen, C. (2004). Very clever homunculus: Compound stimulus strategies for the explicit task-cuing procedure. Psychonomic Bulletin \& Review, 11, 832-840.

MaYr, U., \& Bell, T. (2006). On how to be unpredictable: Evidence from the voluntary task-switching paradigm. Psychological Science, 17, 774-780.

Mayr, U., \& Keele, S. W. (2000). Changing internal constraints on action: The role of backward inhibition. Journal of Experimental Psychology: General, 129, 4-26.

MaYr, U., \& KLIEGL, R. (2003). Differential effects of cue changes and task changes on task-set selection costs. Journal of Experimental Psychology: Learning, Memory, \& Cognition, 29, 362-372.

MeIRAN, N. (1996). Reconfiguration of processing mode prior to task 
performance. Journal of Experimental Psychology: Learning, Memory, \& Cognition, 22, 1423-1442.

Monsell, S. (2003). Task switching. Trends in Cognitive Sciences, 7 , 134-140.

Monsell, S., \& Mizon, G. A. (2006). Can the task-cuing paradigm measure an endogenous task-set reconfiguration process? Journal of Experimental Psychology: Human Perception \& Performance, 32, 493-516.

PHILIPP, A. M., \& КоCH, I. (2006). Task inhibition and task repetition in task switching. European Journal of Cognitive Psychology, 18, 624-639.

Schneider, D. W., \& Logan, G. D. (2006). Priming cue encoding by manipulating transition frequency in explicitly cued task switching. Psychonomic Bulletin \& Review, 13, 145-151.

\section{NOTE}

1. It is worth pointing out that the manipulation of task-switch-2 transitions inversely and uniformly modulates the probability of all remaining transition types (see Table 1). This may compromise the ability to directly compare overall RTs and error scores across the two between-subjects conditions. However, because the relative frequencies of the three remaining transition types is not affected, this does not compromise the ability to interpret modulations of cue-switch costs and actual switch costs.

(Manuscript received July 14, 2005;

revision accepted for publication March 20, 2006.) 